\title{
Controlling the Earth's albedo using reflective hollow glass spheres
}

\author{
Andrew Gordon Neil Walter \\ 4 Austen Avenue, Oliver's Battery, \\ Winchester, Hampshire, SO22 4HP, UK \\ E-mail: a.g.n.walter@exeter.oxon.org
}

\begin{abstract}
Volcanic eruptions have been found to cool the Earth's atmosphere by ejecting dust into the atmosphere. It should be possible to mimic this effect by designing a substitute for this dust consisting of buoyant reflective glass bubbles. This substitute will form a more efficient and long lasting sunscreen. This paper describes the design of such glass bubbles, and the implications and constraints imposed by the choice of materials. It shows the proposal is technically and economically feasible, controllable, of limited lifespan, and environmentally neutral. Actual development and testing will be needed to determine some of the parameters involved. It is clear the idea can be developed and tested experimentally for a very low initial outlay in cost and resources.
\end{abstract}

Keywords: global environmental issues; climate change; geoengineering; albedo; glass bubbles; glass spheres; microspheres; hydrogen; helium; sunscreens.

Reference to this paper should be made as follows: Walter, A.G.N. (2011) 'Controlling the Earth's albedo using reflective hollow glass spheres', Int. J. Global Environmental Issues, Vol. 11, No. 2, pp.91-108.

Biographical notes: Andrew Gordon Neil Walter obtained a degree in Engineering Science at Oxford University. Having worked in Engineering $\mathrm{R} \& \mathrm{D}$ for ten years followed by 26 years at IBM, he is currently retired, and is dedicated to projects that interest him. He is the author of several patents at both places of employment.

\section{Introduction}

Concern exists that climate change is a growing problem. Most industrial nations have targets to reduce greenhouse gas (GHG) emissions in order to reduce man-made effects on climate change. Continuing worldwide industrial development suggests it will be difficult in the short term to reduce or even to stabilise GHG levels. The development of new carbon-neutral technologies has so far been slow, raising the need for interim approaches to the problems of climate change.

Average atmospheric temperature is also subject to variations due to changes in the Sun's output. The Earth's orbit and tilt also vary over a long time-scale, and these cycles also affect the amount of solar radiation reaching Earth (Milankovitch Cycles, undated). 
This paper describes a direct way to control average atmospheric temperature independently of levels of GHGs and solar radiation. However this geoengineering proposal does not address the problem of acidification of the oceans caused by increased $\mathrm{CO}_{2}$ levels.

It is known volcanic eruptions have the side-effect of reducing global temperatures, due to the introduction of aerosols into the stratosphere, which directly scatter sunlight back into space. Harries and Futyan (2006) show the cooling effect lasted for about nine months, by which time the aerosols were effectively flushed from the atmosphere. Temperatures only recovered to normal levels after about two years, suggesting oceanic thermal mass was responsible for a time-lag effect. This pattern of cooling suggests it should be possible to engineer a form of dust, designed to remain in the atmosphere for several years, and to reflect radiation more efficiently than volcanic dust. This would form an efficient and economical sunscreen.

The papers by Teller et al. $(1997,2002,2004)$ describe several methods of albedo control. One of their suggestions includes metallic thin-walled super-pressure balloons, but no detail is given as to method of construction, stresses, or lifetimes. Aluminium is suggested as a possible metal.

Glass bubbles would naturally be much more water-resistant than aluminium, and could possibly be silvered internally or made opaque to achieve an enhanced reflective effect. Correctly designed, these bubbles could be launched from the Earth's surface and still rise to useful altitudes in the troposphere, depending on the stress attainable in the bubble film. Stratospheric altitudes could be achieved with launching made from aircraft or balloons.

Several materials will be suggested in this paper, their engineering suitability discussed, and estimates made of the quantity of materials required.

In order for the bubbles to have a reasonable cost benefit, they need to remain in the atmosphere for longer than the nine months of volcanic aerosols. This implies they need to be more buoyant than solid particles. The total cross-sectional area of all the bubbles should be maximised for the quantity of material used, to reflect as much radiation as possible. The use of vacuum-filled bubbles is superficially attractive, but such bubbles would need to resist buckling under compressive stress. However, the density and strength properties of practical materials mean bubbles able to resist buckling would be too dense to float anywhere in the atmosphere.

\section{Design requirements for albedo control bubbles}

1 the bubbles must be buoyant in the Earth's atmosphere

2 to operate most efficiently, the bubbles should float as high as possible

3 the lifetime of the bubbles should be predictable and controllable

4 failure mechanisms will constrain the lifetime of the bubbles, and need to be determined. Some will be predictable, but many will have to be determined experimentally

5 to be cost-effective, the bubbles should survive in the atmosphere for a long period of time 
6 the bubbles should have maximum cross-sectional area and reflectivity for a given cost in materials, resources and infrastructure

7 the bubbles should be safe and environmentally neutral, during and after their lifetime.

These requirements are now discussed in more detail.

\section{Lifetime}

Bubbles might need to be removed from the atmosphere because:

1 they reflect too much radiation

2 they are found to have undesirable side-effects

3 a change in the Sun's radiation level makes them superfluous

4 volcanic eruptions raise natural dust levels

5 dramatic GHG reductions make them superfluous

6 social or political considerations make them unacceptable.

The lifetime of the bubbles should be compatible with the time-scales of such events. A design life of five years is assumed. The bubbles need to survive ultra-violet (UV) radiation, humidity, temperature and pressure changes. The high levels of UV suggest plastics would not be suitable, leaving metal and glass as more promising candidates. Soda-lime glass is inexpensive, already made in large quantities, relatively inert and can be blown or manipulated to form extremely thin films, so is the material considered in this paper. For strength, titanium would be appropriate, but is difficult to process and likely to be too expensive. Aluminium alloys corrode rapidly, while the pure form has inferior strength. However, pure aluminium has a strength-to-weight ratio similar to glass, so it should not be entirely ruled out of consideration.

\section{Failure mechanisms}

Expected failure mechanisms include:

1 Thermal cycling causing fatigue failure, leading to rupture or cracking and loss of buoyancy, causing the bubble to descend.

2 Temperature changes (sunrise, sunset, cloud-shadowing) may cause pressure changes resulting in rupture or implosion. Reduced pressure will tend to deform a bubble. It remains to be seen to what extent a thin glass film is able to flex under such circumstances, and when shattering will occur. This will be affected by the safety factor in the design and the condition of the film.

3 Up-draughts and down-draughts will take bubbles to unintended altitudes, where they may rupture or implode. At very low altitudes they might collide with high ground. 
4 Contact with water molecules will gradually leach out ions, weakening the glass film, ultimately leading to leaks or rupture and failure.

5 Bubbles may become trapped in ice and rain formation, caught in rainstorms, impacted by planes, hit by lightning or corroded by acids.

Given this non-exclusive list of failure mechanisms it is clear no bubble will survive for ever. They may turn out to have a distribution of lifetimes similar to radioactive isotopes, or maybe a bath-tub curve like electronic components. It is probable the lifetime can only be determined by experience.

\section{Buoyancy}

A glass bubble filled with buoyant gas at local atmospheric pressure can be designed with a film thin enough to make the bubble rise. As it rises, the air-pressure falls, so the bubble becomes less buoyant, but the stress in the glass increases.

For a given film thickness, the altitude to which a bubble can rise is determined by its radius. Smaller bubbles are denser and rise less far, but the stress is lower.

From an understanding of this relationship it is possible to determine an operational altitude for bubbles with a film of a certain thickness, made of a material of known density and strength, filled with a particular gas at a particular pressure.

If the other factors (altitude, stress) are kept constant, the film thickness is always the same fraction of the radius, so larger bubbles require more material for a given total reflective area.

The change in altitude gained by a bubble is independent of the altitude at which it is launched. However, the ratio of film thickness to bubble radius diminishes for bubbles designed to float at higher altitudes.

Hydrogen and helium are the obvious candidates for the buoyant gas. Helium is in limited supply and relatively expensive, but would be adequate for development work. As it is not combustible, using it will improve safety. The quantity of buoyant gas required for full-scale production is likely to exceed global helium supplies, leaving hydrogen as the obvious choice for production, when the bubbles would be manufactured using fully developed dedicated plant. Hydrogen is slightly less dense than helium, but the mass of the gas is much less than the mass of the enclosing film, so the difference in performance would be negligible.

\section{Efficiency}

The higher the bubbles float in the atmosphere, the more solar radiation will be reflected back into space. In this paper the simplifying first-order assumptions are that all incident solar radiation reaches the operational altitude of the bubbles and that the percentage of this radiation incident on the effective reflective area of each bubble is then reflected directly back into space.

Ideally the bubbles should float in the stratosphere, above weather systems, and avoiding most of the water in the atmosphere. The altitude to which the bubbles can be allowed to rise is constrained by the permissible stress in the chosen film material. Lower temperatures at the operating altitude will reduce the internal pressure. At 10,000 m 
above sea-level a drop of around $50^{\circ} \mathrm{C}$ (NRLMSISE, undated) is expected, which will reduce the internal pressure to about $80 \%$ of the pressure at low-altitude launch. The buoyancy gas will gradually permeate through the film, further reducing internal pressure and increasing the lifetime of the bubbles. A high initial stress-level at floating-altitude may therefore be acceptable. The permeability of glass is highly sensitive to its precise composition (Herr and Lercher, 2003), and silvering the bubbles would reduce permeability. Actual permeability and its effect on stress would have to be determined experimentally.

The bubbles in the atmosphere will reflect both the short-wavelength (mostly visible) radiation from the Sun, and the long-wavelength infra-red (IR) radiation being re-radiated back towards space from the Earth's surface. In the latter case the bubbles themselves would behave as a GHG. It would be ideal if the bubbles could be made small enough to become invisible to this IR radiation. Most of the incident short-wavelength solar radiation has a wavelength of less than $2 \mu \mathrm{m}$, while most of the Earth's long-wave IR radiation has a wavelength greater than $5 \mu \mathrm{m}$. There is therefore a gap between the two energy spectra between 2 and $5 \mu \mathrm{m}$. If bubbles can be made with a diameter of 3 to $4 \mu \mathrm{m}$, then they would be 'visible' to the incident radiation, but essentially 'invisible' to the IR. This observation is only useful if it proves possible to use such small-scale bubbles. Calculations in this paper suggest bubble dimensions will be considerably larger in practice.

Bubbles made of pure glass will be highly transparent to the visible radiation from the Sun. An obvious improvement is to silver the bubbles. Teller et al. (1997) state an aluminium film of less than $0.02 \mu \mathrm{m}$ thickness is nearly transparent to the IR radiated from the Earth's surface. This implies a silvered surface of at least this thickness should be provided. The effect of a glass coating may have to be taken into account.

The problem of how to silver these bubbles remains. Suppose the film thickness is to be $0.5 \mu \mathrm{m}$, which is about 60 times the diameter of a single atom of aluminium. Silvering might be achieved by introducing vaporised aluminium into the gas used to fill the bubbles, by a chemical reaction which reduces some of the alumina present in the glass to metallic aluminium, by introducing reflective particles into the glass film material itself, or by exterior silvering. In any case, the effect on buoyancy will be negligible, so the operational altitude would not be affected. A possible alternative to silvering may be to make the bubbles opaque. This might be achieved by doping the glass with suitable materials. Doped glass would provide reflectivity between the two extremes of transparent bubbles and silvered bubbles.

Bubbles might acquire net positive or negative charges, due to friction or photon-induced emission of electrons. This could cause clumping or electrostatic precipitation of atmospheric dust. This in turn might decrease rainfall or increase solar illumination by removing reflecting dust particles from the air. To counteract any such tendencies it might be necessary to deposit a conductive film on the outer surface of the bubbles.

\section{Cost-effectiveness and manufacturing}

The way in which a bubble scatters incident radiation must be considered. For a glossy bubble, the curvature of the surface means incident light will be reflected in a variety of 
directions, ranging from straight back to glancing tangential reflections which have no useful effect. The useful reflections are those which direct radiation away from the Earth's surface. This means only half the cross-sectional area of a bubble should be considered as contributing to the required total percentage coverage of the Earth's surface. This is referred to as the 'effective reflective area' of a bubble. Twice the quantity of bubbles is required than a naïve assumption based on the cross-sectional area would suggest.

The distribution of bubbles over the Earth's surface must also be considered. In order to attempt to reflect $1 \%$ of the radiation arriving at the operating altitude, the aim should be to provide $1 \%$ coverage where the solar radiation is strongest, which is at equatorial areas at mid-day. Achieving this coverage over the lit hemisphere, which has a curved area twice that of the planar cross-section of the Earth, means making twice the quantity of bubbles required to cover $1 \%$ of the Earth's cross-sectional area.

As seen from the Sun, the bubbles will have 1\% coverage at the middle of the Earth's cross-section (where it is mid-day near the equator), but rather more than $1 \%$ coverage at the periphery (where it is typically dawn or dusk). As the light-path through the atmosphere to the peripheral bubbles is considerably greater than the path to the central bubbles, the peripheral bubbles will have much less opportunity to contribute to useful reflections. It is therefore better to specify that the percentage coverage should be understood to apply at the centre of the cross-section. The greater density of peripheral bubbles will be cancelled out to some extent by being less efficient. This means a further factor of twice the quantity is required.

Bubbles must be provided for both the lit hemisphere and the night-time hemisphere of the Earth, introducing a further factor of two.

If one bubble is twice the radius of another, the larger bubble has four times the crosssectional area and can be expected to reflect four times as much radiation. However, the larger bubble requires double the film thickness if operational altitude and film stress are to remain unchanged. It therefore uses eight times as much glass and gas as the smaller bubble. Doubling the dimensions of the bubbles therefore means doubling the material used for the same total reflective capacity.

Conversely, halving the bubble radius and film thickness is likely to reduce the material used by a similar factor. But bubble lifetime is likely to be reduced, because thinner films will fail sooner due to the effect of etching and the leaching of ions by atmospheric moisture. Smaller bubbles will therefore need replacing sooner than larger bubbles. Their lower material cost will not necessarily reduce the total running cost of the system.

The minimum thickness of the silvered layer needs to be approximately $0.02 \mu \mathrm{m}$. This thickness does not need to be increased for larger bubbles. Creating this layer therefore requires a constant volume of aluminium, irrespective of the size of the bubbles. Bubbles which last longer increase the lifetime of this layer, reducing the running costs of the system by reducing the frequency this fixed-volume reflective layer needs to be replaced.

Glass bubbles with diameters ranging from 10 to $200 \mu \mathrm{m}$ and film thickness from 0.5 to $20 \mu \mathrm{m}$ have already been manufactured (Herr and Lercher, 2003) for use in hydrogen storage and transport containers, holding hydrogen under pressures of up to $100 \mathrm{MPa}$. Resisting such high pressures requires a much greater ratio of film thickness to radius than the present scheme requires. The same manufacturing methods may be applicable. These storage bubbles were made by sol-gel processes 
(Shelby and Rapp, 2004-2005) but may not scale efficiently. Larger bubbles might be manufactured by bubbling buoyant gas through films or baths of molten glass, and relying on surface tension to form bubbles of appropriate diameter and film thickness. These might then be separated by fluid jets, ultrasound or mechanical vibrations. There will be manufacturing problems to overcome; newly formed bubbles made in this way might implode as the gas inside will remain hot after the glass film has solidified, causing these bubbles to become squashed as the gas cools. However, thin glass films are remarkably flexible, and if fracture can be avoided, the bubbles might be launched in a deformed state, gradually reverting to spherical form as they rise in the atmosphere.

Unsilvered thin glass films exhibit interference effects. Depending on the exact film thickness and the wavelength of the incident light, 4\% of light incident at 90 degrees to the surface should be reflected from the first face, and $4 \%$ of the remainder should be reflected from the second surface. However, allowing for interference effects, the reflectivity of the film exhibits a sinusoidal variation with frequency, varying from $0 \%$ to $16 \%$ with an average of $8 \%$ reflectivity [Feynman, (1990), Chapter 1, Figure 5]. As the film thickness drops below 0.5 of a wavelength the reflectivity drops towards zero. The $92 \%$ of the light transmitted inside the bubble results in a similar $8 \%$ of $92 \%$ which may be reflected back at the exiting film layer, so a further contribution to overall reflectivity is expected. More detailed calculation or actual measurement is needed, but at least $10 \%$ reflectivity could reasonably be expected. As glass films age, they may develop a natural opalescence which will alter the reflectivity. This effect will also need to be factored in.

To minimise material usage, the film thickness is likely to be of the order of $0.5 \mu \mathrm{m}$ or less, which is of the order of the wavelength of the incident sunshine. Silvered bubbles might approach $80 \%$ reflectivity, which is around eight times the reflectivity expected from plain glass bubbles. Nevertheless, if silvering turns out to be expensive, it may be cheaper to use a larger quantity of unsilvered bubbles.

The operational altitude of the bubbles depends on the film stress; a stress of $100 \times 10^{6} \mathrm{~N} \mathrm{~m}^{-2}$ corresponds to an altitude gain of $1,950 \mathrm{~m}$; a stress of $200 \times 10^{6} \mathrm{~N} \mathrm{~m}^{-2}$ corresponds to an altitude gain of $3,420 \mathrm{~m}$, and $300 \times 10^{6} \mathrm{~N} \mathrm{~m}^{-2}$ corresponds to $4,595 \mathrm{~m}$. Highly stressed bubbles are more likely to fail prematurely, so they should be launched from as high an altitude as possible. Many cities are situated above $3000 \mathrm{~m}$, and locations such as the top of Pike's Peak in the USA at 4,300 m could be used. Much is known about wind patterns, and bubbles launched from appropriate locations should avoid high mountain ranges, which do not exceed 8,848 metres high.

Alternative methods might be used to avoid stress-limited altitude gain, such as:

1 Manufacture bubbles inside aircraft or balloons at high altitude.

2 Enclose bubbles manufactured at ground level but designed for high altitude, in containers at low pressure, and transport these containers to the operating altitude.

3 Manufacture bubbles designed for high altitude at ground-level, and fill them with hydrogen at ambient pressure. These bubbles would rupture if carried directly up to high altitude. But the hydrogen could be partially released in transit by warming the bubbles (Herr and Lercher, 2003) or by using photo-enhanced hydrogen diffusion (Shelby and Rapp, 2004-2005). The hydrogen released might be used to assist in powering the vehicle or helping the release process. 
4 Manufacture the bubbles as in 3, above. Then, heat them at ground-level while reducing the ambient pressure to reduce the internal pressure by hydrogen diffusion. After a return to ambient pressure the bubbles may simply flatten somewhat without shattering. In this state the bubbles could be released, and will rise, gradually resuming spherical shape as they approach the operating altitude.

\section{Environmental factors}

Some bubbles may leak and drift downwards. Bubbles of very small diameter could potentially be breathed in, and the health effects of this should be considered. The density of failing and failed bubbles at ground level is hard to predict, but it should be considerably less than the density of bubbles floating at altitude, which itself will be very low.

Very small particles with dimensions of the order of $2.5 \mu \mathrm{m}$ and $10 \mu \mathrm{m}$ are classified as PM2.5 and PM10 respectively (Staff of Air Resources Board, 2002). There are safety standards associated with exposure to particles of these sizes. In 2002, the Air Resources Board of the California Environment Protection Agency recommended (Staff of Air Resources Board, 2002)

- the PM10 annual-average standard be lowered to $20 \mu \mathrm{g} \mathrm{m}^{-3}$.

- the PM10 24-hour-average standard be retained at $50 \mu \mathrm{g} \mathrm{m}^{-3}$.

- a new PM2.5 annual-average standard be set at $12 \mu \mathrm{g} \mathrm{m}^{-3}$.

- a new PM2.5 24-hour-average standard be set at $25 \mu \mathrm{g} \mathrm{m}^{-3}$.

These concerns suggest bubble diameters should be set well above $10 \mu \mathrm{m}$.

The thinnest practical film is $0.02 \mu \mathrm{m}$. This corresponds to bubble radii between $0.2 \mathrm{~mm}$ and $13 \mathrm{~mm}$. These are considerably larger than PM10 particles and should not pose a health hazard.

Bubbles destroyed in the atmosphere will end up as fine dust, but much of this will gradually be washed out by rain and should pose no health hazard.

Some bubbles will impact aircraft. This may cause increased abrasion to windows and engine components.

Some bubbles will be sucked into jet-engine intakes. The bubbles floating at operational altitude will have the same density as the surrounding air, so mass flow-rate through an engine will be unchanged. The volume of bubbles ingested will be far below the volume of air, so the effect on engine performance will be negligible.

As bubbles die, their hydrogen content will be released into the atmosphere. The rate of release of this gas will depend on the size of the bubbles chosen, and their lifetime. Hydrogen is not an effective GHG, and this release should pose little hazard to the environment. 


\section{Material estimates}

In estimating the material requirements the following bubble characteristics are assumed:

1 Silvered glass bubbles launched from a ground location, filled with hydrogen at ambient atmospheric pressure.

2 Bubbles assumed initially impermeable to hydrogen.

3 Initial stress at operating altitude: $2 \times 10^{8} \mathrm{~N} \mathrm{~m}^{-2}$.

4 Film thickness: $0.5 \mu \mathrm{m}$.

5 Operational altitude: 9,000 m (to clear Mt. Everest at 8,848 m).

6 Effective coverage: $1 \%$ of the Earth's surface. This will not reduce the solar radiation by exactly $1 \%$. The required coverage will need to be determined by climate modelling.

7 Bubble lifetime: five years approximately. This value affects the replacement rate for failed bubbles, and may determine the final value of film thickness once the failure mechanisms are well understood. There is as yet no certainty that bubbles made with a film thickness of $0.5 \mu \mathrm{m}$ will last for five years.

These choices provide enough information to calculate the size and buoyancy of bubbles. Using the formulae (see the Appendix), we get:

1 Altitude gain: 3,423 metres (this is independent of launch altitude and bubble radius, and is limited by the allowable stress at operational altitude).

2 Launch altitude $5,577 \mathrm{~m}$. This is simply the operational altitude minus the altitude gain.

3 Bubble radius: $11 \mathrm{~mm}$. This exceeds the size of the microspheres described in reference (Herr and Lercher, 2003), implying the need for new manufacturing methods. The actual radius given by the formulae is not particularly significant, but it shows clearly that the bubbles will be well above the 'invisible-particle or dust-like' size which might cause respiratory problems.

4 The total film mass for these parameters is 64 million tonnes of glass (see Table 1). If the (yet to be determined) lifetime of the bubbles is five years, this requires 13 million tonnes per annum, which represents $25 \%$ of the current global production of float glass in 2008 [Pilkington, (2009), Section 1.1].

5 The total gas volume required is 89 thousand million cubic metres. This considerably exceeds the world annual production of helium, estimated at 170 million cubic metres (US Government, 2007), reinforcing the choice of hydrogen. 
Table 1 Variables and constants used

\begin{tabular}{|c|c|c|c|}
\hline Name & Units & Description & Value (if constant) \\
\hline$R_{E}$ & $\mathrm{~m}$ & Radius of Earth & $6.378 \times 10^{6}$ \\
\hline$P_{\mathrm{STP}}$ & $\mathrm{N} \mathrm{m}^{-2}$ & $\begin{array}{l}\text { Atmospheric pressure at standard } \\
\text { temperature and pressure (STP) }\end{array}$ & $1.013 \times 10^{5}$ \\
\hline$\rho_{\text {air(STP) }}$ & $\mathrm{kg} \mathrm{m}^{-3}$ & Density of air at STP & 1.293 \\
\hline$\rho_{\mathrm{he}(\mathrm{STP})}$ & $\mathrm{kg} \mathrm{m}^{-3}$ & Density of helium at STP & 0.178 \\
\hline$\rho_{\text {hyd(STP) }}$ & $\mathrm{kg} \mathrm{m}^{-3}$ & Density of hydrogen at STP & 0.090 \\
\hline$\rho_{\text {aluminium }}$ & $\mathrm{kg} \mathrm{m}^{-3}$ & Density of aluminium silvering film & $2.7 \times 10^{3}$ \\
\hline$T_{\text {aluminium }}$ & $\mathrm{m}$ & Thickness of aluminium silvering film & $0.02 \times 10^{-6}$ \\
\hline$R$ & $\mathrm{~m}$ & Radius of bubble & \\
\hline$T$ & $\mathrm{~m}$ & Film thickness of bubble & \\
\hline$E a$ & $\mathrm{~m}^{2}$ & Effective reflective area of bubble & \\
\hline$S$ & Scale $0-1$ & $\begin{array}{l}\text { Silvering coefficient, } 0.0 \text { (transparent) to } \\
1.0 \text { (perfect reflector) }\end{array}$ & \\
\hline$F$ & Scale $0-1$ & $\begin{array}{l}\text { Fraction of Earth's surface area to be } \\
\text { covered by the sum of the effective } \\
\text { reflective area of all bubbles }\end{array}$ & \\
\hline$Z$ & $\mathrm{~m}$ & Altitude above sea level & \\
\hline$Z a$ & $\mathrm{~m}$ & $Z$ at bubble operational altitude & \\
\hline$Z g$ & $\mathrm{~m}$ & $Z$ at ground level (bubble launch altitude) & \\
\hline$P_{\text {air(Z) }}$ & $\mathrm{N} \mathrm{m}^{-2}$ & Atmospheric pressure at altitude $Z$ & \\
\hline$P_{i}$ & $\mathrm{~N} \mathrm{~m}^{-2}$ & Internal pressure inside bubble & \\
\hline$\rho_{\text {film }}$ & $\mathrm{kg} \mathrm{m}^{-3}$ & Density of film material & $2.5 \times 10^{3}$ for glass \\
\hline$\rho_{\text {air(Z) }}$ & $\mathrm{kg} \mathrm{m}^{-3}$ & Density of air at altitude $Z$ & \\
\hline$\rho_{\mathrm{gas}(\mathrm{pi})}$ & $\mathrm{kg} \mathrm{m}^{-3}$ & $\begin{array}{c}\text { Density of buoyant gas at internal } \\
\text { pressure } P_{i}\end{array}$ & \\
\hline$M_{b}$ & $\mathrm{~kg}$ & Mass of bubble & \\
\hline$\sigma_{D}$ & $\mathrm{~N} \mathrm{~m}^{-2}$ & $\begin{array}{c}\text { Film design-stress (less than yield stress } \\
\text { by some safety factor) }\end{array}$ & \\
\hline Tfm & $\mathrm{kg}$ & Total film mass of all bubbles deployed & \\
\hline Tsm & $\mathrm{kg}$ & $\begin{array}{l}\text { Total silvering mass of all bubbles } \\
\text { deployed }\end{array}$ & \\
\hline $\operatorname{Tg} v$ & $\mathrm{~m}$ & $\begin{array}{c}\text { Total gas volume (at STP) of all bubbles } \\
\text { deployed }\end{array}$ & \\
\hline
\end{tabular}

There is the potential to reduce the film thickness of the bubbles below this example. Reducing the film thickness from to $0.5 \mu \mathrm{m}$ to $0.25 \mu \mathrm{m}$ would halve the glass and gas used. Reducing the film thickness to $0.1 \mu \mathrm{m}$ would reduce the glass required to 13 million tonnes. However, the lifetime of the bubbles will certainly be reduced, so annual turnover of bubbles will be greater, possibly negating the apparent savings. 
The total silvering mass $T_{s m}$ is purely dependent on the required thickness, the fractional coverage $F$ of Earth's surface, and the silvering coefficient $S$. Making this thickness the minimum able to reflect solar photons, minimises the materials used, for reflective bubbles suspended in the atmosphere acting as conventional mirrors.

Similarly, the total film mass $T f m$ is purely dependent on its thickness $T$, fractional coverage $F$ and the actual silvering coefficient $S$.

The tonnage of film material which needs to be operating in the atmosphere is linear with the film thickness of the bubbles, and independent of their radius. If glass is used to make these then the tonnage of glass required could lie anywhere from 64 million tonnes down to as little as 6.4 million tonnes. This represents a glass tonnage from just over one year's global output down to possibly as little as two month's output, but the continuous actual annual requirement will be highly dependent on the lifetime, which drives the replacement rate. This assumes coverage of $1 \%$ of the Earth's surface area with bubbles of $80 \%$ silvering reflectivity.

The tonnage of reflective aluminium film needed to be operating in the atmosphere is constant, estimated to be approximately 2.8 million tonnes. Annual requirements are similarly driven by the lifetime of the bubbles. Again, these figures will need adjustment to match actual coverage and reflectivity.

The volume of buoyancy gas needed to be operating in the atmosphere is linear with the film thickness of the bubbles. Annual requirements are similarly driven by the lifetime of the bubbles, as well as coverage and reflectivity.

Table 1 lists the variables and constants used in this paper and in the Appendix, and their units.

Tables 2 and 3 list the bubble dimensions and material tonnages, for a range of desired operational height gains, launch heights, and stress levels.

Table 2 Altitude gain, bubble radius, tonnage of film material, and gas volume as a function of stress, launch altitude, film thickness

\begin{tabular}{lcccccc}
\hline$\sigma_{D}\left(\mathrm{~N} \mathrm{~m}^{-2}\right)$ & $Z_{g}(\mathrm{~m})$ & $T(\mathrm{~m})$ & $Z a-Z g(\mathrm{~m})$ & $R(\mathrm{~m})$ & $T f m(\mathrm{~kg})$ & $\operatorname{Tgv}\left(\mathrm{m}^{3}\right)$ \\
\hline $1 \times 10^{8}$ & 1,000 & $0.5 \times 10^{-6}$ & 1,951 & $4.8 \times 10^{-3}$ & $64 \times 10^{9}$ & $71 \times 10^{9}$ \\
$2 \times 10^{8}$ & 1,000 & $0.5 \times 10^{-6}$ & 3,423 & $6.0 \times 10^{-3}$ & $64 \times 10^{9}$ & $89 \times 10^{9}$ \\
$3 \times 10^{8}$ & 1,000 & $0.5 \times 10^{-6}$ & 4,596 & $7.2 \times 10^{-3}$ & $64 \times 10^{9}$ & $107 \times 10^{9}$ \\
$1 \times 10^{8}$ & 3,000 & $0.5 \times 10^{-6}$ & 1,951 & $6.3 \times 10^{-3}$ & $64 \times 10^{9}$ & $71 \times 10^{9}$ \\
$2 \times 10^{8}$ & 3,000 & $0.5 \times 10^{-6}$ & 3,423 & $7.9 \times 10^{-3}$ & $64 \times 10^{9}$ & $89 \times 10^{9}$ \\
$3 \times 10^{8}$ & 3,000 & $0.5 \times 10^{-6}$ & 4,596 & $9.5 \times 10^{-3}$ & $64 \times 10^{9}$ & $107 \times 10^{9}$ \\
$1 \times 10^{8}$ & 5,000 & $0.5 \times 10^{-6}$ & 1,951 & $8.3 \times 10^{-3}$ & $64 \times 10^{9}$ & $71 \times 10^{9}$ \\
$2 \times 10^{8}$ & 5,000 & $0.5 \times 10^{-6}$ & 3,423 & $10.4 \times 10^{-3}$ & $64 \times 10^{9}$ & $89 \times 10^{9}$ \\
$3 \times 10^{8}$ & 5,000 & $0.5 \times 10^{-6}$ & 4,596 & $12.6 \times 10^{-3}$ & $64 \times 10^{9}$ & $107 \times 10^{9}$ \\
\hline
\end{tabular}

Notes: Film thickness set constant at: $T=0.5 \mu \mathrm{m}$.

Fractional coverage set constant at: $F=0.01$.

Silvering coefficient set constant at: $S=0.8$.

Mass of the reflective silvered film and effect on buoyancy and stress are ignored.

For this value of $T$, the total silvering mass is constant.

$T_{s m}=2.8 \times 10^{9} \mathrm{~kg}=2.8$ million tonnes. 
Table 3 Altitude gain, bubble radius, tonnage of film material, gas volume as a function of stress, launch altitude, film thickness

\begin{tabular}{lcccccc}
\hline$\sigma_{D}\left(\mathrm{~N} \mathrm{~m}^{-2}\right)$ & $Z_{g}(\mathrm{~m})$ & $T(\mathrm{~m})$ & $Z a-Z g(\mathrm{~m})$ & $R(\mathrm{~m})$ & $T f m(\mathrm{~kg})$ & $T g v\left(\mathrm{~m}^{3}\right)$ \\
\hline $1 \times 10^{8}$ & 1,000 & $0.05 \times 10^{-6}$ & 1,951 & $0.48 \times 10^{-3}$ & $6.4 \times 10^{9}$ & $7.1 \times 10^{9}$ \\
$2 \times 10^{8}$ & 1,000 & $0.05 \times 10^{-6}$ & 3,423 & $0.60 \times 10^{-3}$ & $6.4 \times 10^{9}$ & $8.9 \times 10^{9}$ \\
$3 \times 10^{8}$ & 1,000 & $0.05 \times 10^{-6}$ & 4,596 & $0.72 \times 10^{-3}$ & $6.4 \times 10^{9}$ & $10.7 \times 10^{9}$ \\
$1 \times 10^{8}$ & 3,000 & $0.05 \times 10^{-6}$ & 1,951 & $0.63 \times 10^{-3}$ & $6.4 \times 10^{9}$ & $7.1 \times 10^{9}$ \\
$2 \times 10^{8}$ & 3,000 & $0.05 \times 10^{-6}$ & 3,423 & $0.79 \times 10^{-3}$ & $6.4 \times 10^{9}$ & $8.9 \times 10^{9}$ \\
$3 \times 10^{8}$ & 3,000 & $0.05 \times 10^{-6}$ & 4,596 & $0.95 \times 10^{-3}$ & $6.4 \times 10^{9}$ & $10.7 \times 10^{9}$ \\
$1 \times 10^{8}$ & 5,000 & $0.05 \times 10^{-6}$ & 1,951 & $0.83 \times 10^{-3}$ & $6.4 \times 10^{9}$ & $7.1 \times 10^{9}$ \\
$2 \times 10^{8}$ & 5,000 & $0.05 \times 10^{-6}$ & 3,423 & $1.04 \times 10^{-3}$ & $6.4 \times 10^{9}$ & $8.9 \times 10^{9}$ \\
$3 \times 10^{8}$ & 5,000 & $0.05 \times 10^{-6}$ & 4,596 & $1.26 \times 10^{-3}$ & $6.4 \times 10^{9}$ & $10.7 \times 10^{9}$ \\
\hline
\end{tabular}

Notes: Film thickness set constant at: $T=0.05 \mu \mathrm{m}$.

Fractional coverage set constant at: $F=0.01$.

Silvering coefficient set constant at: $S=0.8$.

Mass of the reflective silvered film and effect on buoyancy and stress are ignored. For this value of $T$, the total silvering mass is constant.

$T_{s m}=2.8 \times 10^{9} \mathrm{~kg}=2.8$ million tonnes.

The principal difference between these two very similar tables is that Table 3 uses a film thickness one tenth that of Table 2, while keeping the choices of altitudes and stress the same. The formulae used to derive these tables are detailed in the Appendix. Comparing these two tables illustrates several interesting features:

1 the total mass of glass required is a linear function of film thickness, and unaffected by chosen altitudes and stress levels

2 the volume of hydrogen needed is a linear function of film thickness, and also increases with the desired altitude-gain

3 altitude gain is controlled by allowable stress levels only, and is independent of the film thickness and launch altitude chosen

4 the radius of the bubble is not particularly significant, except to note that they will be considerably larger than dust particles and so should not cause respiratory problems associated with breathing dust

5 the total mass of aluminium for silvering is the same in both tables, and is unaffected by any variations of stress, film thickness, altitudes and radius.

\section{Conclusions}

It should be feasible to control climate change by distributing thin-filmed reflective glass spheres into the Earth's atmosphere. Much research needs to be done, so precise material requirements and costs cannot be estimated yet. The principle can be established by small-scale experimentation which could potentially be done for very little initial outlay. 


\section{Questions arising}

A number of questions immediately arise, concerning the social and financial requirements for implementing this proposal. While it is too early to decide these questions, some consideration can be given to them.

1 How long would it take to prove the initial concept?

2 If successful, how long would it take to put into mass production?

3 How would the project be controlled?

4 How would the project be financed?

5 Who would implement the project?

6 How would the launch-sites be determined?

7 How would the atmospheric effects be monitored?

Here are some aspects which should be considered when deciding the questions above.

1 The concept itself is simple, so it should be possible to construct prototype bubbles in quantities of hundreds or thousands, helium-filled and unsilvered for safety and simplicity, within a period of about one to two years. Whether these would be largely hand-crafted (manually by expert glass blowers) or machine-made (maybe by adapting machinery used to make lightbulbs) or by adapting the sol-gel process cannot yet be decided. It would be advisable to attempt several different approaches simultaneously and see which looks most promising. Construction of a few hundred or thousand sample bubbles would be sufficient to determine the optical reflectivity of the bubbles, and it would also be possible to expose them to simulated release into the atmosphere by releasing some into controlled atmospheres such as the geodesic domes at the Eden Project in Cornwall UK. Further prototyping would be needed to develop techniques to silver the bubbles or make them otherwise more reflective or opaque, as well as research into large-scale efficient manufacturing methods. This would take a further three to four years, so totalling approximately five years of work.

2 Assuming success of the prototyping stage, mass production would take considerably longer. The tonnages of glass required are substantial, so it would be a case of ramping up supplies over a period of five to ten years. Melting and forming glass requires high temperatures and a lot of energy, so supplies of electricity may need to be installed. Whether bubbles should be launched from the factory, or transported elsewhere for hydrogen-filling and later launch should be determined by the total cost of each approach, and this will not be known until the prototyping stage has been completed. If multiple different manufacturers of bubbles are used, they may have different technologies which take different times to install and ramp up.

3 The project will need to be run on a basis of international collaboration, and as such would need to be controlled by an organisation such as the United Nations Framework Convention on Climate Change, known as UNFCCC. Many, maybe all the countries of the world, will have an interest in just how the climate is governed. Some countries may favour gentle climate warming as a way to increase crop yields and to make cold areas more habitable, and to ease oil exploration in the Arctic 
regions for example; others which are already very hot, or believe that current warming may be making them even hotter and subject to abnormal weather conditions, may prefer to see average temperatures reduced gradually. There will be a lot of debate, and hard decisions to be made, and there must be a world-wide forum or similar means to reach global agreement on how this project would be managed.

4 Finance will need to be raised, again on an international basis, maybe along the lines of the Carbon taxes levied on industries which generate a lot of GHGs. This proposal does not negate the need for carbon taxing; such taxes may be needed to encourage industry to develop 'green' or carbon-neutral technologies. When atmospheric levels of GHGs are reduced, then the need to launch bubbles should also reduce. This glass bubbles idea simply attempts to reduce the greenhouse-warming effect of GHGs, and should not be seen as a way to avoid responsibility for reducing GHG production. Note that this glass bubbles proposal does not address the problem of increasing ocean acidification; reduction of atmospheric $\mathrm{CO}_{2}$ is the ideal solution for this issue.

5 The project will need a variety of implementers, ranging from materials suppliers, manufacturers of the bubbles themselves, to climate analysts advising on rates of bubble launching and required lifetimes, and analysts monitoring the actual atmospheric performance of the bubbles. A cooperative effort will need to be coordinated, probably by the controlling body.

6 The choice of launch sites will be governed by the need to minimise the total running costs of the project. Using launch sites at higher altitudes is beneficial for the lifetimes of the bubbles, as it means less altitude gain is required to reach the same operating altitude, and this in turn means the bubbles are under less stress and so will last longer. However, it may be more expensive to transport materials and to source the necessary energy supplies at higher altitudes, so these drawbacks need to be balanced. In addition, it may be possible to develop methods which result in bubbles being partially inflated with hydrogen at sea level; if so, such bubbles would be able to achieve much greater altitude gains before reaching the allowable stress levels. This would permit launching bubbles from any location at all, providing a much greater choice of locations. So it will be necessary to complete the prototyping stage before any firm decisions can be made about the final choice of launch sites.

7 It is likely that a variety of different manufacturers will end up making bubbles, some in the northern hemisphere, others in the southern hemisphere, and quite possibly using different manufacturing techniques and slightly different glass compounds. As all these bubbles will end up intermixed in the atmosphere, there will need to be a regular sampling process to evaluate the actual persistence and lifetimes of the different versions of bubbles. This might be done by unmanned drones, or aeroplanes specially adapted to capture bubbles as they fly at different altitudes in different places.

It should be possible to do some land-based monitoring, by shining lasers at lunar corner-reflectors and monitoring the amount of scattering of the reflected light, or by monitoring the scattering of sunlight at dusk, but this form of monitoring may not be able to distinguish the separate contributions made by different versions of bubbles, making actual high altitude sampling necessary. 


\section{References}

Feynman, R.P. (1990) QED: The Strange Theory of Light and Matter, Penguin, London, ISBN 0-140-1-25051.

Harries, J.E. and Futyan, J.M. (2006) 'On the stability of the Earth's radiative energy balance: response to the Mt. Pinaturbo eruption', Geophysical Research Letters, Vol. 33, L23814, doi: $10.1029 / 2006$ GL027457.

Herr, M. and Lercher, A. (2003) 'Hydrogen storage in microspheres', available at http://www.esa.int/gsp/completed/030910ESTEC16292.pdf (accessed on 4 September 2010).

Milankovitch Cycles (undated) Available at http://en.wikipedia.org/wiki/Milankovitch_cycles (accessed on 4 September 2010).

NRLMSISE (undated) 'NRLMSISE standard atmosphere model', available at $\mathrm{http} / / /$ en.wikipedia.org/wiki/NRLMSISE-00 (accessed on 4 September 2010).

Pilkington (2009) 'Pilkington and the flat glass industry', available at http://www.pilkington.com/resources/pfgi2009final.pdf (accessed on 4 September 2010).

Schott (2007) 'Schott technical glasses', available at http://www.schott.com/epackaging/english/download/english_technical_gl ass_handbook.pdf (accessed on 4 September 2010).

Shelby, J. and Rapp, D. (2004-2005) 'Photo-enhanced hydrogen diffusion through glass microspheres: development of microsphere production for hydrogen storage', available at http://ceer.alfred.edu/Research/glassdiffusion.html (accessed on 4 September 2010).

Staff of Air Resources Board (2002) 'Public hearing to consider amendments to the ambient air quality standards for particulate matter and sulfates', available at $\mathrm{ftp} / / / \mathrm{ftp}$.arb.ca.gov/carbis/research/aaqs/std-rs/pm-final/exesum.pdf (accessed on 4 September 2010).

Teller, E., Hyde, R. and Wood, L. (2002) 'Active climate stabilization: practical physics-based approaches to prevention of climate change', available at http://www.osti.gov/accomplishments/documents/fullText/ACC0233.pdf (accessed on 4 September 2010).

Teller, E., Hyde, R., Ishikawa, M., Nuckolls, J. and Wood, L. (2004) 'Active climate stabilization: presently-feasible albedo-control approaches to prevention of both types of climate change', Proc. Symp. on Macro-Engineering Options for Climate Change Management and Mitigation, Tyndall Centre/Isaac Newton Institute, Cambridge University, Cambridge, UK.

Teller, E., Wood, L. and Hyde, R. (1997) 'Global warming and ice ages: 1. Prospects for physicsbased modulation of global change', available at http://dge.stanford.edu/labs/caldeiralab/Caldeira downloads/Teller_etal_LLNL231636_ 1997.pdf (accessed on 4 September 2010).

US Government (2007) HELIUM, available at http://minerals.usgs.gov/minerals/pubs/commodity/helium/heliumcs07.pdf (accessed on 4 September 2010). 


\section{Appendix}

Details of formulae used in the paper

\section{Formulae}

These apply to a bubble filled with gas at launch altitude $Z g$ above sea-level such that the internal pressure $=$ local atmospheric pressure which is assumed to follow a straight-line approximation to the NRLMSISE graph (NRLMSISE, undated). Surface tension effects are ignored, and the stress in the film is assumed to be zero when the bubbles are filled at ground level. The bubble is then assumed to be released to float in equilibrium at design altitude $Z a$ above sea-level. At this altitude the stress in the film is the design-stress $\sigma_{D}$. In practice temperature drops should reduce this stress level by up to $20 \%$, slightly increasing any safety margin. Loss of buoyancy gas due to diffusion will subsequently reduce the stress to safe long-term levels. Loss of buoyancy gas will also reduce the bubble mass, increasing the operational altitude, but this increase is small, of the order of 180 metres, so is ignored as being unimportant.

\begin{tabular}{|c|c|}
\hline Formula & Description \\
\hline$E a=\pi \mathrm{R}^{2} \mathrm{~S} / 2$ & Effective reflective area of a single bubble \\
\hline$P_{\operatorname{air}(\mathrm{Z})}=P_{S T P} \mathrm{e}^{(-Z / 7,238)}$ & Pressure of atmosphere at altitude $Z$ \\
\hline$\rho_{\operatorname{air}(\mathrm{Z})}=\rho_{\operatorname{air}(\mathrm{STP})} \mathrm{e}^{(-Z / 7,238)}$ & Density of atmosphere at altitude $Z$ \\
\hline$\rho_{\mathrm{gas}(\mathrm{Z})}=\rho_{\mathrm{gas}(\mathrm{STP})} \mathrm{e}^{(-Z / 7,238)}$ & Density of gas at altitude $Z$ \\
\hline$M_{b}=4 \pi \mathrm{R}^{2} T \rho_{\text {film }}+(4 / 3) \pi \mathrm{R}^{3} \rho_{\mathrm{gas}(\mathrm{pi})}$ & Mass of a single bubble \\
\hline$M_{b} \leq(4 / 3) \pi \mathrm{R}^{3} \rho_{\text {air }(\mathrm{Z})}$ & Condition for buoyancy \\
\hline$T \geq R\left(P_{i}-P_{\text {air }(\mathrm{Z})}\right) / 2 \sigma_{D}$ & Stress condition for film to resist rupture \\
\hline$T=R P_{S T P}\left(\mathrm{e}^{(-Z g / 7,238)}-\mathrm{e}^{(-Z a / 7,238)}\right) / 2 \sigma_{D}$ & $\begin{array}{l}\text { Film thickness of bubble floating in } \\
\text { equilibrium at altitude } Z a\end{array}$ \\
\hline \multirow{2}{*}{$Z a-Z g=7,238 \ln _{e}\left\{\frac{\left(\sigma_{D} \rho_{\mathrm{air}(\mathrm{STP})}+1.5 P_{S T P} \rho_{\text {film }}\right)}{\left(\sigma_{D} \rho_{\mathrm{gas}(\mathrm{STP})}+1.5 P_{S T P} \rho_{\text {film }}\right)}\right\}$} & \multirow[t]{2}{*}{ Altitude gain for a bubble after launch } \\
\hline & \\
\hline$T f m=32 \pi \mathrm{R}_{E}^{2} F T \rho_{f i l m} / S$ & Total film mass of all bubbles \\
\hline $\operatorname{Tg} v=\left(32 \pi \mathrm{R}_{E}^{2} F \operatorname{Re}^{(-Z g / 7,238)}\right) / 3 S$ & Total gas volume at STP of all bubbles \\
\hline$T s m=32 \pi \mathrm{R}_{E}^{2} F T_{\text {aluminium }} \rho_{\text {aluminium }} / S$ & Total mass of silvering layer \\
\hline
\end{tabular}

\section{Derivation of formulae}

1 Derivation of $E a$ : consider a bubble above the equator with sunshine incident from vertically above. Reflected radiation is required to escape back into space. Any portion of the bubble's film sloped at more than 45 degrees from horizontal will simply deflect radiation to ground. This performs no useful purpose. The effective area is the uppermost section of the bubble which slopes at less than 45 degrees to the horizontal. Similarly, consider this bubble at sunset. Solar radiation striking the top hemisphere will be reflected in a useful spaceward direction, and the same formula applies. Whether this formula is correct at all other angles of incidence is yet 
to be shown, but it is adequate for the first-order approximate calculations in this paper.

2 Derivation of $P_{\text {air(Z): }}$ : See reference (NRLMSISE, undated) where pressure at sea-level $=1$ atmosphere $=1.013$ Bar. Fit a straight-line to the logarithmic-linear graph such that at $50 \mathrm{Km}$ elevation above sea-level, the pressure is assumed to have reduced by a factor of 1,000 . An equation of the form

- $\quad P=A e^{(-B Z)}$ can be solved given two pressures. For the small altitude-changes used in this paper, the ambient temperature is assumed to be unchanged at all altitudes.

- $\quad \rho_{\text {air(Z) }}$ Follows directly from $P V / T=$ constant, assuming the gas to be 'ideal' and temperature remains constant.

3 Derivation of $M_{b}$ : a bubble is modelled as a thin-filmed sphere where $\mathrm{T} \ll \mathrm{R}$.

4 Derivation of stress condition: consider a hemisphere of a bubble. Tensile force resisting bursting is $2 \pi R T \sigma$. Net force on cross-section attempting to separate hemispheres is $\pi \mathrm{R}^{2}\left(P_{i}-P_{\operatorname{air}(\mathrm{Z})}\right)$. These balance, and $\sigma \leq \sigma_{D}$ is required in order not to exceed design-stress.

5 Derivation of buoyancy condition: the mass of air displaced by a bubble is given by:

$$
M_{\text {air }}=(4 / 3) \pi \mathrm{R}^{3} \rho_{\text {air(Z) }}
$$

Buoyancy requires the mass of the bubble to be less than mass of air displaced. This leads to

$$
M_{\text {air }}=M_{b}
$$

for a bubble floating in equilibrium.

6 Derivation of $Z a-Z g$ : take the condition for buoyancy, and the stress condition. Make these into equalities for the situation where a bubble is in equilibrium with the stress at $\sigma_{D}$. The internal gas is assumed to be at the launch-altitude atmospheric pressure, so $\rho_{\mathrm{gas}(\mathrm{pi})}=\rho_{\mathrm{gas}(\mathrm{Zg})}$, and $P_{i}=P_{Z g}$. Eliminate $T$ between these two equations. This gives:

$$
\sigma_{D}\left(\rho_{\mathrm{air}(\mathrm{Za})}-\rho_{\mathrm{gas}(\mathrm{Zg})}\right)=1.5\left(P_{\operatorname{gas}(\mathrm{Zg})}-\rho_{\mathrm{air}(\mathrm{Za})}\right) \rho_{\text {film }} .
$$

Substituting the formulae for $\rho_{\text {air(Z) }}=\rho_{\text {air(STP })} \mathrm{e}^{(-Z / 7,238)}$ and $\rho_{\operatorname{gas}(Z)}=\rho_{\text {gas (STP })} \mathrm{e}^{(-Z / 7,238)}$ using $Z=Z a$ and $Z=Z g$ as appropriate, re-arranging and taking logs to base $e$, gives the final result.

7 Derivation of $T f m$ : surface area of Earth $=4 \pi \mathrm{R}_{E}^{2} F$ and cross-sectional area is $\pi \mathrm{R}_{E}^{2}$. The Sun's radiation is constantly incident on only one hemisphere of Earth. Given the target to create a fraction $F$ of this cross-sectional area in the form of bubbles, this needs a reflective area totalling $\pi \mathrm{R}_{E}^{2} F$ for the hemisphere in sunshine, and $\pi \mathrm{R}_{E}^{2} F$ for the hemisphere in shadow, totalling $2 \pi \mathrm{R}_{E}^{2} F$. Assuming a uniform distribution of bubbles, most of these bubbles will be reflecting radiation not from directly overhead, but from shallow angles (close to dawn and dusk). A more 
conservative estimate would be to achieve the desired $F$ fractional coverage at all times of day and night, so this paper uses $4 \pi \mathrm{R}_{E}^{2} F$ instead. This means the Earth, as seen from space, will have $F$ fractional coverage in the central region where solar radiation arrives 'head-on', and a greater than $F$ coverage at the periphery. Radiation arriving at peripheral bubbles will have passed through a much greater distance of atmosphere, and so stands a much higher probability of being absorbed in the atmosphere before even arriving at these bubbles. The greater density of peripheral bubbles will therefore be cancelled-out to some extent by their being less efficient. The efficiency of a bubble is also proportional to the silvering coefficient $S$. The number of bubbles required is therefore $4 \pi \mathrm{R}_{E}^{2} F / E a S=8 \mathrm{R}_{E}^{2} F / \mathrm{R}^{2} S$. The mass of the film of a bubble is $M_{\text {film }}=4 \pi \mathrm{R}^{2} T \rho_{\text {film }}$. The result follows from multiplying these.

8 Derivation of $T g v$ : the gas mass of a bubble is $M_{g a s}=(4 / 3) \pi \mathrm{R}^{3} \rho_{\mathrm{gas}(\mathrm{pi})}$ (see formula for $M_{b}$ above). Multiplying by the number of bubbles required leads to the total gas mass. Mass $=$ Volume $*$ Density, and substituting to express the pressure of the gas as a function of Launch altitude and pressure at STP leads to the result.

9 Derivation of $T$ : this follows from taking the stress condition and expressing it as an equality, then substituting to represent pressures as a function of launch altitude and floating altitude.

\section{Film stress considerations}

Schott (2007) in Section 3.2 notes that glass has a high theoretical strength in excess of $1 \times 10^{10} \mathrm{~N} \mathrm{~m}^{-2}$ which in practice is limited by surface defects caused by wear to $2 \times 10^{8} \mathrm{~N} \mathrm{~m}^{-2}$. The bubbles should be substantially perfect and free from surface defects, being launched almost immediately after manufacture. It should not be over optimistic to expect the bubbles to survive a stress in the region of 2 to $3 \times 10^{8} \mathrm{~N} \mathrm{~m}^{-2}$ as they arrive at their designed floating altitude, relying on diffusion to reduce this to more sustainable stress-levels. Schott (2007) in Figure 13 shows a reduction factor of 0.3 in constant stress increasing the lifetime of a glass component from seconds to several years. A larger stress reduction could be expected, as diffusion reduces the internal pressure to slightly above the external ambient pressure. 